\title{
Should all women with a history of OASI have a mediolateral episiotomy at their subsequent delivery?
}

\author{
No, this should not be a universal \\ recommendation. Women with a history of obstetric \\ anal sphincter injury (OASI) should be counseled regarding \\ the risks of recurrence, potential risk factors such as \\ macrosomia and increased length of the second stage of \\ labor, and potential mediating factors such as mediolateral \\ episiotomy (MLE) and cesarean delivery. The risks of both \\ episiotomy and cesarean delivery need to be considered, as \\ well as how the existing literature on this topic applies to the \\ specific clinician and patient population involved.
}

\section{$\overline{\text { FAST }}$}

TRACK

For women with

a history of OASI, standardized

recommendations

for mode of delivery

and use of MLE

currently are not

available
Van Bavel J, Ravelli AC, Abu-Hanna A, et al. Risk factors for the recurrence of obstetrical anal sphincter injury and the role of a mediolateral episiotomy: an analysis of a national registry. BJOG. 2020;127:951-956.

\section{EXPERT COMMENTARY}

Jaimey M. Pauli, MD, is Associate Professor, Pennsylvania State College of Medicine, Chief, Division of Maternal-Fetal Medicine, Milton S. Hershey Medical Center, Hershey, Pennsylvania. She serves on the OBG MANAGEMENT Board of Editors.

W omen with a history of OASI are at increased risk for recurrence in a subsequent delivery. Higher rates of anal and fecal incontinence are reported in women with recurrent OASI (rOASI) compared with women who had an OASI only in their first delivery. Previous studies have reported recurrence rates of $5 \%$ to $7 \%,{ }^{1}$ and some suggested that MLE may be protective, but standardized recommendations for mode of delivery and use of MLE currently are not available.

The author reports no financial relationships relevant to this article.
Recently, van Bavel and colleagues sought to determine the rate of rOASI in their population as well as the factors that increase and decrease the risk of this complication.

\section{Details of the study}

This cohort study used data from the Dutch Perinatal Registry (Perined) that included 268,607 women who had their first and second deliveries (singleton, term, vertex, $<43$ weeks) vaginally in 2000-2009. The study's primary objective was to determine the rate of rOASI in women who had OASI in their first delivery. The secondary objectives were to identify risk factors for rOASI and to assess the effect of MLE. For the purposes of this study, OASI was defined as subtotal and total rupture of the perineum, or grades 3A-4 as defined by the Royal College of Obstetricians and Gynaecologists. ${ }^{2}$

Within this cohort, 9,943 women had an OASI in their first delivery (4\%), and the rate of rOASI was $5.8 \%$ ( 579 of 9,943 ). After multivariate analysis, the risk factors for rOASI were birth weight of $4,000 \mathrm{~g}$ or greater (odds ratio [OR], 2.1; 95\% confidence 
interval [CI], 1.6-2.6) and duration of the second stage of labor of 30 minutes or longer (OR, 1.8; 95\% CI, 1.4-2.3).

The MLE rate was $40.8 \%(4,054$ of 9,943$)$ and was associated with a lower rate of rOASI (OR, 0.3; 95\% CI, 0.3-0.4). This association persisted when delivery type was separated into spontaneous and operative vaginal deliveries, with the number of MLEs needed to prevent one rOASI of 22 and 8 , respectively. Birth weight of less than 3,000 g also was noted to be protective against rOASI (OR, 0.5; 95\% CI, 0.3-0.9).

Based on these findings, as well as comparisons to previous studies, the authors concluded that MLE could be considered for routine use or at least discussed with all women with a prior OASI for prevention of rOASI.

\section{Study strengths and limitations}

A strength of this study was the large number of deliveries and the wide variation of practice included in the registry database, which promotes the generalizability of the results and reduces bias. This also provides an adequate base on which to determine an accurate rate of rOASI in the Dutch population.

One study limitation is that information is not available regarding how the episiotomies were performed (specifically, angle of incision), delivery techniques ("hands on" vs "hands off"), and indication for the episiotomy. Additional limitations suggested are that

\section{WHAT THIS EVIDENCE MEANS FOR PRACTICE}

Prevention of rOASI is important, as fecal incontinence is debilitating and difficult to treat. While this study provides evidence that MLE may protect against this complication, its results may not be generalizable to all patient or clinician populations. Differences in baseline rate of MLE and cesarean delivery, technique, indication, and comfort with repair-all not evaluated in this study-must be taken into account when counseling OASI patients about their options for delivery and the use of MLE in a subsequent pregnancy.

JAIMEY M. PAULI, MD

clinicians who perform an episiotomy may have an inherent bias regarding the protective nature of the procedure and may miss a rOASI due to inadequate examination postprocedure, overestimating its protective effect.

Finally, the relatively high rate of MLE and low rate of cesarean delivery $(6.9 \%)$ in this study are specific to the Netherlands and do not reflect the obstetric practices used in many other countries. Generalizability of these results in the context of much lower MLE and higher cesarean delivery rates (as in the United States) would therefore be in question.

Reference

1. Van Bavel J, Ravelli AC, Abu-Hanna A, et al. Risk factors for the recurrence of obstetrical anal sphincter injury and the role of a mediolateral episiotomy: an analysis of a national registry. BJOG. 2020;127:951-956.

2. Royal College of Obstetricians and Gynaecologists. Green top guideline No. 29: the management of third- and fourthdegree perineal tears. June 2014. https://www.rcog.org.uk /globalassets/documents/guidelines/gtg-29.pdf. Accessed June 12, 2020. 